\title{
Using Coded Excitation to maintain Signal to Noise for FMC+TFM on Attenuating Materials
}

\author{
David Lines, Ehsan Mohseni, Yashar Javadi, Carmelo Mineo, Randika Vithanage, Zhen Qiu, Charles MacLeod, Gareth Pierce, Anthony \\ Gachagan \\ Centre for Ultrasonic Engineering \\ University of Strathclyde \\ Glasgow, UK \\ david.lines@strath.ac.uk
}

\begin{abstract}
Ultrasonic Non-Destructive Evaluation using Full Matrix Capture (FMC) and Total Focusing Method (TFM) is used for high resolution imaging as every pixel is in optimal focus. FMC excites one element in turn, so operates with lower transmitted energy compared to phased array beamforming. The energy at a reflector is further reduced by the broad directivity pattern of the single element. The large number of $\mathrm{Tx} / \mathrm{Rx} \mathrm{A}$-scans that contribute to each pixel recover the Signal-to-Noise Ratio (SNR) in the final TFM image. Maintaining this in the presence of attenuating materials is a challenge because relevant information in each Ascan signal is buried in the thermal noise, and the TFM process assumes no quantization effects in the Analogue-to-Digital Converters (ADCs) in each receiver. In-process inspection during Additive Manufacturing (AM) requires ultrasonic array sensors that can tolerate high temperatures, scan over rough surfaces and leave no residue. Dry-coupled wheel probes are a solution, but the tire rubbers are often highly attenuating, causing a problem for FMC+TFM needed to adapt the focus through the rough surface. Common approaches to maintain the SNR are to drop the frequency or to average over multiple transmissions, but these compromise resolution and acquisition rate respectively. In this paper, the application of coded excitation to maintain the SNR in the presence of high signal attenuation is explored.
\end{abstract}

Keywords-Ultrasound, Coded excitation, orthogonal Golay code, FMC, TFM, high attenuation, additive manufacturing

\section{INTRODUCTION}

It is highly desirable to do in-process ultrasonic inspection during Additive Manufacturing (AM) as this will allow immediate corrective action, including repair of any defects and adjustment of process parameters $[1,2]$. The challenges include inspecting at elevated temperatures, without leaving a residue, through an unknown, irregular interface that will distort the direction and focus of the ultrasound beams. The first two constraints are being addressed by using a dry-coupled, high temperature roller array probe [3], but the available rubber tires are highly attenuating compared with the AM material.

Ultrasonic Non-Destructive Evaluation (NDE) using Full Matrix Capture (FMC) and Total Focusing Method (TFM) is used for high resolution imaging because every pixel is in optimal focus [4]. A further benefit when used for in-process $\mathrm{AM}$ inspection is that the beamforming is implemented as a post-processing operation [5], which allows adaptive compensation (ATFM) for unknown and irregular interfaces [6, 7,8 ], addressing the remaining challenge.

FMC excites one element of the array in turn, and so operates with lower transmitted energy compared to phased array beamforming. The energy arriving at a reflector is further reduced, compared with that formed from multiple transmit elements, by the broad directivity pattern of the single element. The Signal-to-Noise Ratio (SNR) in the final TFM image is recovered by summing the contributions from the large number of Transmit (Tx)/Receive ( $\mathrm{Rx})$ A-scan pairs. Maintaining the SNR in the presence of attenuating materials is a challenge because relevant information in each A-scan signal is buried in the thermal noise. The reconstruction process assumes no quantization or saturation effects in the Analogue-to-Digital Converters (ADCs) in each $\mathrm{Rx}$ channel. This requires each to have a high dynamic range to avoid saturation on large echoes whilst correctly recovering the defect echoes from within the noise. Ensuring the channel filters are matched to the transducer bandwidth minimises noise, but the most common approaches to maintain the SNR in difficult applications are to drop the ultrasound array frequency or to average over multiple transmissions, but these compromise resolution and acquisition rate respectively.

Radar and sonar use coded excitation to extend the dynamic range without compromising resolution and these techniques have also been applied to ultrasound imaging [9, 10, 11]. Excitation using complementary Golay pairs allows long sequences, for best SNR improvement, and matched filtering with minimal processing artifacts. It also offers the potential for multiple simultaneous transmissions using mutually-orthogonal pairs $[12,13,14]$. The rest of this paper reports on the use of Golay-coded excitation to maintain the SNR of FMC acquisition, such as through attenuating tire materials for inprocess AM inspection using FMC+ATFM.

\section{BACKGROUND}

Complementary pairs of Golay codes are generated by the recursive algorithm in [15], and so have lengths of $2^{\mathrm{N}}$ cycles where $\mathrm{N}$ is an integer with minimum value of 1 . An ultrasonic acquisition is modified by replacing each single transmission 
with firing the first of the pair (TxA) and then the second of the pair (TxB), thereby doubling the number of transmissions. The received signals ( $\mathrm{RxA} \& \mathrm{RxB}$ ) are cross-correlated with the corresponding excitation and the results summed. The special property of the complementary Golay pair is that the processing sidelobes exactly cancel, as shown in Fig. 1 for 16 cycles.

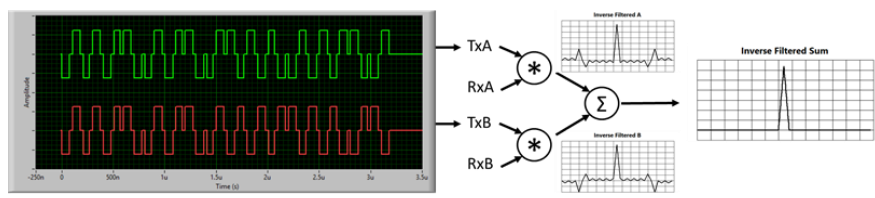

Fig. 1. 16 cycle Golay pair processing showing sidelobe cancellation

Averaging over a pair of A-scans produces an SNR improvement of $3 \mathrm{~dB}$ as the noise is random. Every doubling of the excitation length also produces a $3 \mathrm{~dB}$ improvement in SNR [16]. For a Golay pair excitation with each having $2^{\mathrm{N}}$ cycles, a net SNR improvement of $3(\mathrm{~N}+1) \mathrm{dB}$ is expected, compared to a single cycle. The resulting A-scan is normalised to the reference of single-cycle, single-excitation, and so the noise floor will be reduced by $3(\mathrm{~N}+1) \mathrm{dB}$.

\section{EXPERIMENTAL RESULTS}

A Phased Array Controller (PAC) with tri-level excitation capability (FIToolbox ${ }^{\circledR}$ from Diagnostic Sonar Ltd., Livingston, $\mathrm{UK},[17])$ was used to collect FMC data from a $10 \mathrm{MHz} 32$ element $0.31 \mathrm{~mm}$ pitch array (Olympus 10L32-9.9x10-A31-P2.5-HY) on an ASTM E2491 Phased Array Assessment Block (1018 Steel). The system was setup with the array in direct contact on the block, using SoundClear ${ }^{\circledR}$ couplant (Diagnostic Sonar Ltd., Livingston, UK).

\section{A. Verification of SNR improvement}

The aim of the first experiment was to verify the SNR improvement as the Golay code length increases. The system was operated in FMC mode, with the array coupled to the flat surface, as shown in Fig. 2 (left). The maximum response from the backwall is obtained when the same element is used on Tx as well as $\mathrm{Rx}$, so the elements along the FMC diagonal were monitored and the largest displayed. A small echo, with low $\mathrm{SNR}$, is needed to mimic the condition of FMC through attenuating materials. A low excitation voltage $(+/-10 \mathrm{~V})$ was therefore used and the receive gain adjusted until the first backwall repeat echo was around $50 \%$ of display height. The resulting A-scan, with fixed gain of $58 \mathrm{~dB}$, is shown in Fig. 2 (right).
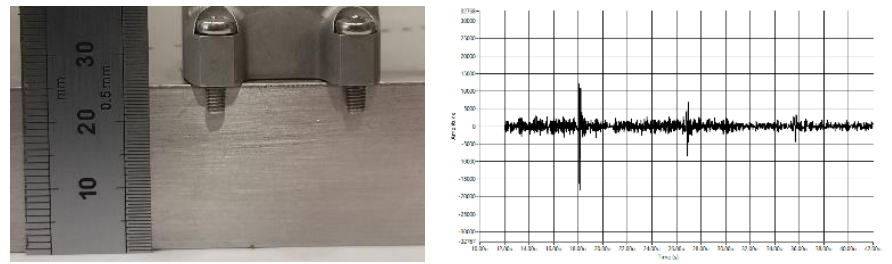

Fig. 2. Acquisition setup for SNR verification (left), and the resulting Ascan showing $1^{\text {st }}, 2^{\text {nd }}$ and $3^{\text {rd }}$ backwall repeat echoes (right).

The dynamic range of the echo was measured over a range of acquisition modes, starting with the reference of single cycle excitation. The second case was with same setup but averaged over 2 pulses. The remaining modes were with Golay acquisition and processing for 2, 4, 8, 16, 32 and 64 cycles in turn. In each case, the RMS noise was measured with the same setup but with the transmitters disabled and the SNR calculated as the ratio between measured echo amplitude and RMS noise. The results are shown in Fig. 3, with the trend confirming the expected $3 \mathrm{~dB}$ improvement between each step.

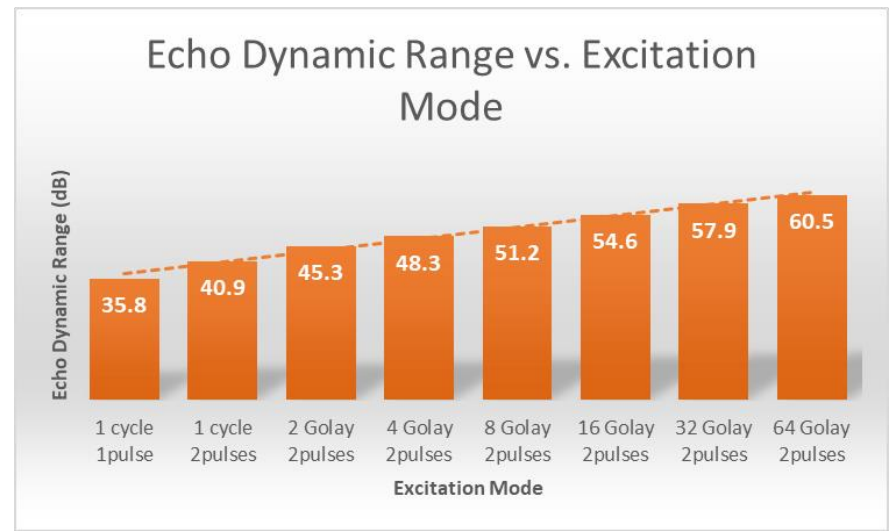

Fig. 3. Measured echo dynamic range vs. Golay excitation length.

Fig. 4 shows the A-scans for a 16 cycle Golay excitation (left) and after processing (right) with its complementary pair.
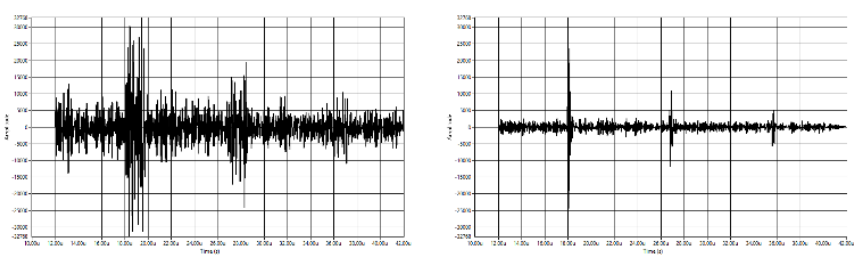

Fig. 4. A-scans of 16 cycle Golay before (left) and after processing (right).

Comparing the latter with Fig. 2 (right) confirms that the temporal resolution has been restored to the same as the single cycle excitation.

\section{B. FMC + TFM imaging through attenuating material}

Reference FMC data was collected using single cycle excitation with a $5 \mathrm{MHz} 32$ element $0.5 \mathrm{~mm}$ pitch array directly coupled to the top surface of the block, to image the middle region of both quadrants of side-drilled holes (SDHs), and a $60 \mathrm{~mm} \times 60 \mathrm{~mm}$ TFM image produced, Fig. 5 (left).

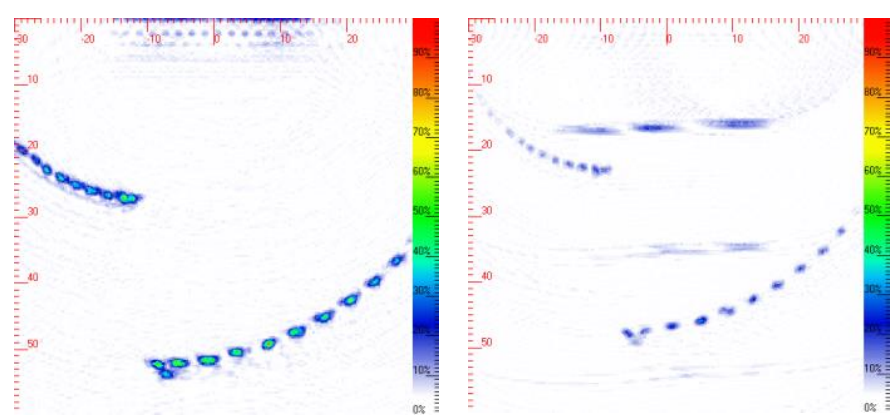

Fig. 5. TFM results from direct contact (left) and with $3 \mathrm{~mm}$ rubber (right). 
Samples of $3 \mathrm{~mm}$ and $6 \mathrm{~mm}$ thick high temperature silicone rubber (Shore A20 from Primasil), typical of the high temperature materials investigated for the roller probe tire, were then inserted between array and block, as in Fig. 6. Couplant was used on both sides of the rubber and a new set of FMC data acquired, and a new TFM image produced, Fig 5 (right), for the material beyond the rubber/steel interface.

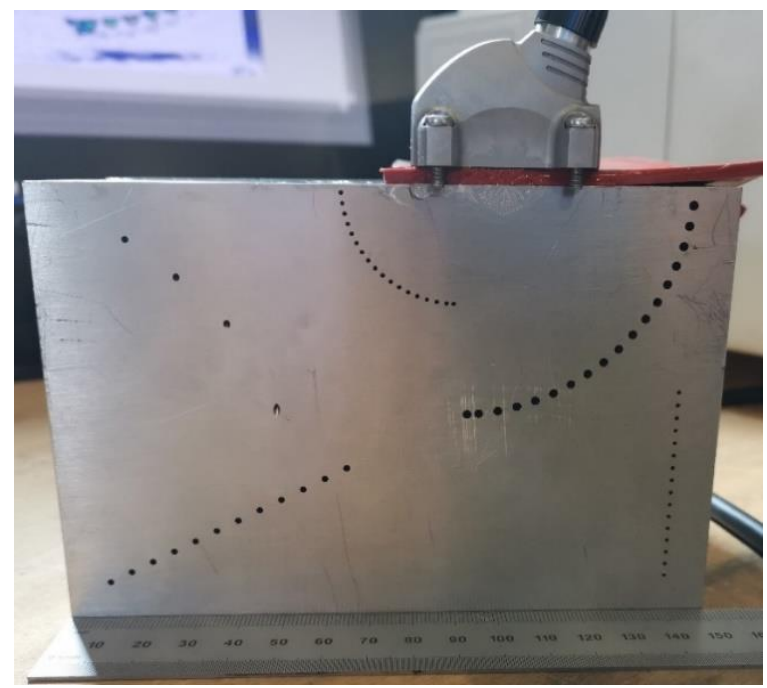

Fig. 6. $5 \mathrm{MHz}$ acquisition setup with $6 \mathrm{~mm}$ rubber between array and block.

The attenuation in the rubber was measured using a throughtransmission technique in a tank to be $0.7 \mathrm{~dB} / \mathrm{mm}$ at $5 \mathrm{MHz}$ so the round-trip attenuation is $4.2 \mathrm{~dB}$ for $3 \mathrm{~mm}$ material and $8.4 \mathrm{~dB}$ for $6 \mathrm{~mm}$. In the contact data, the gain was increased to the maximum before any of the FMC A-scans saturated, satisfying the linear criteria for TFM reconstruction. The strongest echo with the $3 \mathrm{~mm}$ rubber is from the rubber/steel interface and acquiring the single cycle data with the gain maximized without this echo saturating resulted in the largest SDH echo being around $20 \mathrm{~dB}$ down compared to the contact case. Instead, the TFM image was monitored as the gain was further increased, until the first rubber repeat echo started distorting, producing the image in Fig. 6 (right). Normalising the display so that the SDH echoes are the same amplitude as in Fig. 5 (left) required a further $9.5 \mathrm{~dB}$ gain. This increased the noise to an unacceptable level, confirming the need for increased dynamic range

The AM interface geometry requires dry-coupling via a thicker tire and $6 \mathrm{~mm}$ is preferred, both of which increase the attenuation. The defect size to be detected means an increase to $10 \mathrm{MHz}$ is also desirable, further compounding the signal loss. The $10 \mathrm{MHz} 32$ element array had couplant between array and rubber but this was dry-coupled to the block to image the smaller quadrant of SDHs. FMC data was collected using 8 cycle Golay pairs with the gain increased by $24 \mathrm{~dB}$ over that used in Fig. 5 (right) but without saturation. The resulting $40 \mathrm{~mm} \times 40 \mathrm{~mm}$ TFM image in Fig. 7 shows the near field SDHs are correctly represented without loss of axial resolution. Despite the additional $6 \mathrm{~dB}$ of post-processing gain to normalise the SDH amplitude, the image still had good contrast without noise. 8 cycles was chosen over 16 or 32 cycles as it still gave acceptable
SNR and ensured the dead zone after transmission didn't compromise the near field performance.

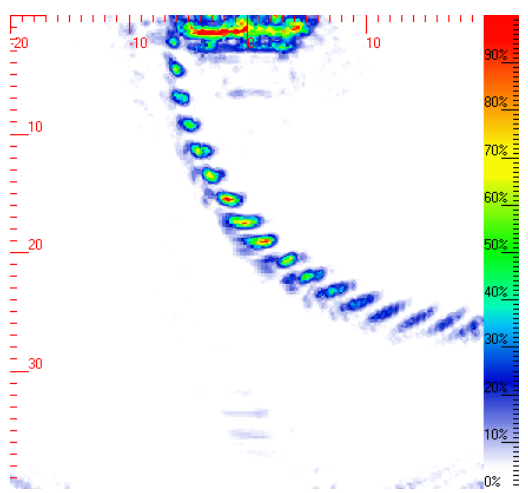

Fig. 7. $10 \mathrm{MHz}$ TFM through $6 \mathrm{~mm}$ dry-coupled rubber.

\section{Orthogonal code excitation}

The above results have confirmed the viability of coded excitation to increase the dynamic range, sufficient to use $10 \mathrm{MHz}$ through a $6 \mathrm{~mm}$ dry-coupled high temperature rubber tire. Achieving a similar SNR to the 8 cycle Golay pair by averaging would have taken $16 \mathrm{x}$ as long as the single cycle reference and $8 \mathrm{x}$ that of the Golay pair acquisition. This comparison is even worse for the cases where the SNR requires longer Golay excitations, but there is still the desire to operate as fast as possible. Simultaneous transmission from pairs of elements with orthogonal Golay codes means that the transmission signals can be separated out on reception by the matched inverse filtering, offering the potential to recover the $2 \mathrm{x}$ loss in speed.

The $5 \mathrm{MHz} 64$ element $0.5 \mathrm{~mm}$ pitch array was used with couplant directly on the surface of the block to collect FMC data. The $32 \mathrm{~mm} \times 32 \mathrm{~mm}$ image of the linear sequence of SDHs produced from 64 standard 16 cycle Golay pair transmissions (128 pulses in total) is shown in Fig. 8 (left). The matching result from 64 orthogonal simultaneous pair excitations (64 pulses in total), is shown in Fig.8 (right), confirming the validity of this technique.

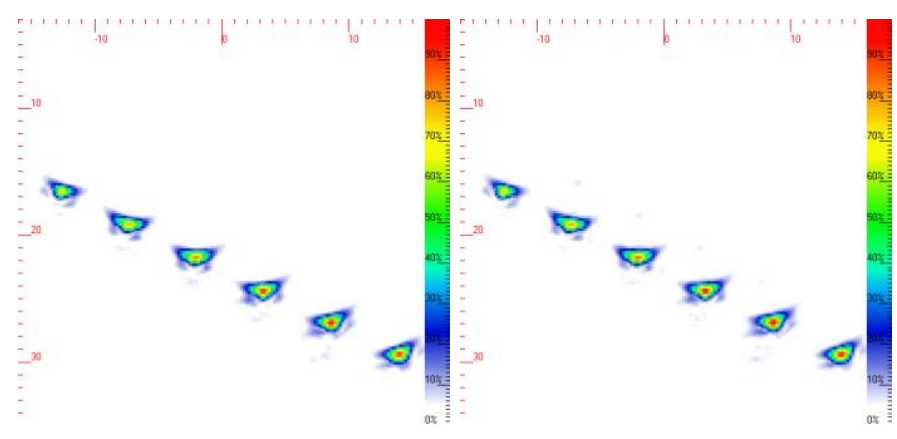

Fig. 8. 128 pulse standard Golay (left) and 64 pulse orthogonl pairs (right).

This FMC data set used elements offset by half the length of the array, to highlight if there was any cross-coupling between orthogonal pulses. However, the same clean result was observed when firing adjacent elements and stepping by 2 . 


\section{DISCUSSION AND CONCLUSIONS}

Coded excitation techniques are used in other fields but the research in ultrasound applications has been targeted at medical with limited NDE use. The approach can offer $10 \mathrm{~s}$ of $\mathrm{dB}$ improvements in SNR without compromising range resolution and with limited impact on acquisition time - using orthogonal codes means no impact. Whilst processing time is increased, this could be done offline. However, a commercial implementation is more likely to have the processing done within the Field Programmable Gate Array (FPGA) electronics commonly used in the instrument front end [17].

A disadvantage of long pulse excitation is the increase in the post-transmission dead zone before the first receive signals are detected. Many applications use an acoustic wedge or delay line, so this is not an issue other than imposing an upper limit on the pulse length. Dynamic range is rarely an issue in the extreme near field, so shorter code lengths can be chosen for the application, with the limit being single cycle transmission.

$\mathrm{FMC}+\mathrm{TFM}$ is a growing technique, especially for in-process AM inspection where adaptive TFM will be necessary, and here the SNR improvement may be essential because of the attenuation through the roller probe's dry-coupled tire. However, coded excitation could also benefit other NDE applications, including: avoiding the need to drop frequency, and resolution, in difficult materials such as austenitic steels and Glass Fiber Reinforced Plastic; allowing processing of the highly attenuated off-axis beams in Carbon Fiber Reinforced Plastic; and operation in air-coupled inspection roles [18].

These are areas where existing approaches are challenged, but the technique could also be used for existing applications. PACSs have pulsers that typically operate up to $200 \mathrm{~V}$, a limit set by the integrated circuit technology. Operation at $12 \mathrm{~V}$ would mean the pulsers could be integrated with the other front end electronics and also avoid the need for high voltage power supplies, but would drop the received signal by $24 \mathrm{~dB}$. This loss could be recovered by use of coded excitation. If current inspections can use $100 \mathrm{~V}$ or lower, the PAC could now be miniaturized into a single chip design with $3.3 \mathrm{~V}$ excitation, addressing intrinsically safe as well as conventional applications.

The results presented confirm that Golay code transmission and processing can achieve the predicted benefits in SNR without compromising range resolution or acquisition time.

\section{ACKNOWLEDGMENT}

The high temperature roller probe work was funded by the EPSRC via the RCNDE. Thanks are also due to Grant Smiley for the attenuation measurements on the high temperature rubbers.

\section{REFERENCES}

[1] Y. Javadi, et al., "Ultrasonic phased array inspection of wire plus arc additive manufacture (WAAM) samples using conventional and total focusing method (TFM) imaging approaches", INSIGHT, vol. 61, no. 3, pp. 145-148, March 2019.

[2] Y. Javadi et al., "Ultrasonic phased array inspection of a Wire + Arc Additive Manufactured (WAAM) sample with intentionally embedded defects," Additive Manufacturing, vol. 9, October 2019.
[3] C. Macleod et al, "Dry-coupled automated inspection for wire + arc additive manufacture," Proceedings of QNDE Conference, July 2019.

[4] C. Holmes, B. W. Drinkwater, and P. D. Wilcox, "Post-processing of the full matrix of ultrasonic transmit-receive array data for non-destructive evaluation," NDT \& E International, vol. 38, no. 8, pp. 701-711, December 2005.

[5] D. Lines, "Rapid distributed data collection with arrays - the next step beyond full waveform capture," INSIGHT, vol 48, no. 2, pp. 84-88, February 2006.

[6] M. Parrilla, J. Brizuela, J. Camacho, A. Ibáñez, P. Nevado, and C. Fritsch, "Dynamic focusing through arbitrary geometry interfaces," IEEE Ultrasonics Symposium, pp. 1195-1198, November 2008.

[7] L. Le Jeune, S. Robert, P. Dumas, A. Membre and C. Prada, "Adaptive ultrasonic imaging with the Total Focusing Method for inspection of complex components immersed in water," Proceedings of QNDE Conference, July 2014.

[8] J. Zhang, B. W. Drinkwater, and P. D. Wilcox, "Efficient immersion imaging of components with nonplanar surfaces," IEEE Transactions on Ultrasonics, Ferroelectrics, and Frequency Control, vol. 61, no. 8, August 2014

[9] M. Odonnell, "Coded Excitation System for Improving the Penetration of Real-Time Phased-Array Imaging-Systems," IEEE Transactions on Ultrasonics Ferroelectrics and Frequency Control, vol. 39, no. 3, pp. 341351, May 1992.

[10] R. Y. Chiao and X. H. Hao, "Coded excitation for diagnostic ultrasound: A system developer's perspective," IEEE Transactions on Ultrasonics Ferroelectrics and Frequency Control, vol. 52, no. 2, pp. 160-170, February 2005.

[11] J. A. Isla and F. B. Cegla, "Simultaneous transmission and reception on all elements of an array: binary code excitation," Proceedings of The Royal Society A -Mmathematical Physical and Eengineering Sciences, vol. 475, no. 2225, April 2019.

[12] I. Trots, "Mutually orthogonal Golay complementary sequences in synthetic aperture imaging systems," Archives of Acoustics, vol. 40, no. 2, pp. 283-289, 2015

[13] I. Trots, A. Nowicki, and M. Postema, "Ultrasound image improvement by code bit elongation," IEEE Signal Processing Letters, vol. 25, no. 3, pp. 437-441, Mar. 2018.

[14] F. F. Zhao and J. W. Luo, "Diverging wave compounding with spatiotemporal encoding using orthogonal Golay pairs for high frame rate imaging," Ultrasonics, vol. 89, pp. 155-165, Sep. 2018.

[15] X. Huang, "Complementary properties of Hadamard Matrices," Proceedings of International Conference on Communications, Circuits and Systems, pp.588-592, Jun 2006.

[16] I. A. Veres, A. Cleary, G. Thursby, C McKee, I. Armstrong, S.G. Pierce, and B. Culshaw, "Golay code modulation in low-power laser-ultrasound," Ultrasonics, vol 53, pp.122-129, April 2013.

[17] D. Lines, J.Wharrie and J. Hottenroth, "Real-time full matrix capture + total focusing and other novel imaging options using general purpose PCbased array instrumentation," INSIGHT, vol. 54, no. 2, pp. 86-90, February 2012.

[18] R. Su, D. Lines, Y. Arnaudov, L. Kang, A. Jäger, H. Wang, S.N. Ramadas, M. Kupnik, and S. Dixon "Coded Excitation for Air-Coupled Ultrasound Phased Arrays," Proceedings of NDT2017, Telford, September 2017. 